\title{
Influence of dopamine deficiency in early Parkinson's disease on the slow stimulation multifocal-ERG
}

\author{
Anja M. Palmowski-Wolfe ${ }^{1}$, Maria Timoner Perez ${ }^{1,2}$, Stefanie Behnke ${ }^{2}$, Gerhard Fuss ${ }^{2}$, \\ Martin Martziniak $^{2}$ \& Klaus W. Ruprecht ${ }^{1}$ \\ ${ }^{1}$ Department of Ophthalmology, University of Saarland, D 66421, Homburg, Germany; ${ }^{2}$ Department of \\ Neurology, University of Saarland, D 66421, Homburg, Germany
}

Accepted: 8 February 2006

Key words: dopamine, mfERG, m-sequence, multifocal stimulation techniques, Parkinson

\begin{abstract}
Purpose: In animal studies intravitreal injection of tetrodotoxin (TTX) results in mfERG waveform changes similar to those observed in glaucoma. As TTX blocks amacrine as well as ganglion cells, there is still a question regarding the underlying cell population responsible for these changes in waveform. In an attempt to assess the contribution of the amacrine cells to these changes, a mfERG was obtained from patients with Parkinson's disease as some amacrine cells are mediated by dopamine, a substance lacking in Parkinson's. Methods: Eight patients with early Parkinson's disease underwent ophthalmologic examination, testing of contrast sensitivity and electrophysiological examination according to ISCEV standard at least $12 \mathrm{~h}$ following their last medication with Dopamine. A slow stimulation mfERG was obtained with a stimulus base interval of $53.3 \mathrm{~ms}$ and with a stimulus base interval of $106.6 \mathrm{~ms}$. During MF-ERG recordings 103 hexagons stimulated the central $50 \mathrm{deg}$ of the retina simultaneously and independently (msequence $2^{13}, \mathrm{~L}_{\max }: 200 \mathrm{~cd} / \mathrm{m}^{2}, \sim 100 \%$ contrast). Results: Contrast sensitivity and ISCEV standard electrophysiological testing was unremarkable. When the mfERG was analyzed, only four patients had an adequate signal-to-noise ratio to allow further data analysis - one of whom was diagnosed with a multi system atrophy in retrospect. The first order response component was analyzed at a filter setting of $10-$ $300 \mathrm{~Hz}$ and at 100-300 Hz (OPs) and compared to mfERGs of a control group. On average, in patients, the amplitude of N1P1 was slightly lower in the central and nasal response averages. When the three OPs at a latency of 72-89 ms were analyzed in the $53.3 \mathrm{~ms}$ base interval recording, the most marked difference in amplitude was observed in the superior nasal response average of the first OP. Here a mean amplitude of $1.3 \mathrm{nV} / \mathrm{deg}^{2}$ in patients compared to a mean amplitude of $1.9 \mathrm{nV} / \mathrm{deg}^{2}$ in the control group (P: 0.08 ). Discussion: In contrast to our previous findings in NTG, there was a consistent presence of three OPs. Under the stimulus conditions applied, we did not find an influence of dopaminergic amacrine cells on the mfERG in our patients with moderate stages of Parkinsion's. The difficulties in obtaining an adequate signal-to noise ratio due to e.g. muscle artifacts even in Parkinson patients of moderate disease stages render a success of mfERG recording in patients with more advanced stages unlikely. The question of the influence of dopaminergic amacrine cells on the mfERG could possibly be addressed using MPDT in animal research.
\end{abstract}

Presented in part at the Meeting of the International Society for Clinical Electrophysiology of Vision, Nagoya, Japan, 2003.

Supported by DFG Pa 609/2. 


\section{Introduction}

The multifocal electroretinogram (mfERG) allows topographic mapping of retinal function. It has been applied to analyze the function of different retinal layers such as the outer [1-5] or inner [6] retina.

Most research applying the mfERG to test inner retinal function has been conducted in glaucoma, a disease that poses a major threat to vision in industrial countries. In glaucoma an inner retinal component, the so called optic nerve head component (ONHC), may be distinguished from a more outer retinal component (RC) [7]. The ONHC is a nonlinear mfERG response component whose propagation seems dependent on the nerve fiber layer and which is attributed mainly to the ganglion cell layer [7-12]. It appears to be diminished in glaucoma [7, 13].

Recently a special slow mfERG stimulus sequence has been suggested to be sensitive in OAG [14, 15]. With an increase in the stimulus base interval to $53.3 \mathrm{~ms}$ or more, there is no more overlap between the induced component and the m-sequence response. Under these conditions, oscillatory potentials become apparent in the induced component [16, 17]. These mfOPs, show a marked naso-temporal asymmetry [16, 17] which may be attributed to the misalignment and partial cancellation of the $\mathrm{RC}$ with the ONHC in the nasal retina and their relative alignment and enhancement in the temporal retina [16]. Under these conditions the sensitivity to detect normal tension OAG increases to about $85 \%$ [15].

The oscillatory potentials of the photopic ERG receive a strong contribution from the inner retinal layers [18]. Glycine, GABA and TTX suppress the function of the inner retina and result in reduced or missing oscillatory potentials of the photopic ERG [19]. In mfERG recordings these substances also affect nonlinear contributions to the mfERG $[12,20]$.

In animal studies, mfERG waveforms similar to those observed in glaucoma have been reported following intravitreal injection of TTX. In the slow mfERG TTX decreased the Ops and largely eliminated their nasotemporal waveform asymmetries [17]. TTX blocks some amacrine as well as ganglion cells and interplexiform cells.
Therefore, the cellular origin of the mfERG changes observed in glaucoma or following TTX are still unclear and cannot be attributed exclusively to ganglion cells [21]. As some amacrine cells are dopaminergic, Parkinson's disease would be a potential model to gain further insight into the cellular origin of these mfERG waveform changes.

Parkinson's disease is not only a motor system disease. There is also a concurrent loss of retinal dopamine [22]. This has resulted in a spatial frequency dependent loss of sensitivity for the pattern ERG (PERG) [23, 24] as well as in abnormal pattern visual evoked potentials (PVEP), presumably secondary to retinal changes $[25,26]$. An increase in contrast sensitivity threshold has also been reported in Parkinson's disease [23, 27]. It has been suggested that dopamine has a push-pull effect in the primate retina resulting in a tuned spatial response function [21]. The electrooculogram (EOG) has been reported to show the earliest signs of retinal dysfunction in the very early stages of Parkinson's disease [28] and colour vision deficiencies have been reported to progress during this disease [29].

Thus, in this pilot study an attempt was made to assess the contribution of dopaminergic amacrine cells on the slow stimulation mfERG in patients with Parkinson's disease following at least $12 \mathrm{~h}$ dopamine depletion.

\section{Patients and methods}

Patients with an early stage of Parkinson's disease (clinical presentation as Hoehn and Yahr stages I to II, that is with uni- or bilateral symptoms without alteration of balance) were recruited from the Department of Neurology. Following informed consent, eight patients volunteered to participate in the testing of visual function following a period of at least $12 \mathrm{~h}$ of dopamine deficiency. (Dopamine intake was discontinued for $>12 \mathrm{~h}$ independent of this study in order to evaluate a patient's response to a one time dose of L-dopa.) Exclusion criteria were a history of ocular surgery, ocular diseases, especially glaucoma or diabetic retinopathy as well as high refractive errors $+/-6 \mathrm{dpt}$. A morphologic 
ophthalmic examination followed the testing of visual function described below, to rule out ophthalmic disorders that may affect the results. The patients' right eye was examined, except in one patient where the right eye had a posterior capsular fibrosis following cataract surgery. In this patient, the left eye was included in the study.

Patients underwent testing of EDTRS visual acuity, testing of contrast sensitivity using the Pelli Robson Chart as well as testing of colour vision with the help of the saturated and desaturated panel D15 test. Goldmann perimetry was obtained to rule out visual field defects. A PERG was obtained with a checkerboard check size of $60^{\prime}$ as well as $90^{\prime}$. Further electrophysiology (Ganzfeld ERG, EOG and PVEP) was performed according to ISCEV standard.

Following these examinations patients underwent mfERG recordings as follows:

In all subjects mfERGs were recorded of one eye, which in control subjects was chosen at random, using VERIS ${ }^{\mathrm{TM}}$. MfERG signals were recorded monocularly using a Burian-Allen bipolar contact lens electrode. Pupils were dilated. The viewing distance was adjusted according to the optimal refraction used at a viewing distance of $40 \mathrm{~cm}$ to ensure a constant image size [30].

During recording, the central $50 \mathrm{deg}$ of the retina were stimulated by 103 hexagons where each hexagon flickered according to a slow m-sequence stimulation. Patients underwent two recordings that lasted $7 \mathrm{~min} 17 \mathrm{~s}$ each.

For the first mfERG recording, each m-sequence step (M) with a luminance of either $100 \mathrm{~cd} / \mathrm{m}^{2}$ or $<1 \mathrm{~cd} / \mathrm{m}^{2}$ was followed by 7 black frames (B) with a luminance $<1 \mathrm{~cd} / \mathrm{m}^{2}$. This eight frame stimulus sequence (MBBBBBBB) reoccurred every $106.6 \mathrm{~ms}$. The length of the m-sequence was $2^{12}-1$. The second mfERG recording differed in that only three black frames followed an m-sequence step (MBBB) resulting in a stimulus base interval of $53.3 \mathrm{~ms}$. The length of the m-sequence was $2^{13}-1$.

To enhance the signal-quality each recording was split into 16 cycles of about 27.29 s. The raw signals were filtered $(10-300 \mathrm{~Hz})$ and amplified (gain $=100000$ ). A sampling point was obtained every $0.83 \mathrm{~ms}$. An artifact elimination technique [31] was applied once. The first order response component (KI) was analyzed. For each location $\mathrm{KI}$ is calculated as the difference between the mean local response to all the bright m-sequence stimuli and the mean focal response to the black m-sequence stimuli occurring in a stimulus cycle and taking into account the entire stimulus base interval.

\section{Results}

Mean age of the patients was 62.5 (SD 9.9) years. All patients tested were in an early stage of Parkinson's disease (Schwab scale $>80$ ), that is they could still cope with every day aspects of life, taking twice as long as healthy people to perform these tasks and being aware of this handicap.

On the EDTRS visual acuity chart patients could on average read 60 letters (SD 11). This corresponds to an acuity equivalent of 20/32.

Contrast sensitivity as tested with the Pelli Robson Chart was not impaired. On average patient could achieve Pelli Robson Scores of 1.56 (SD 0.2) which compared to 1.67 (SD 0.06) in a healthy control group.

On colour vision testing four patients had significant abnormalities with the desaturated panel D15 colour plates, consisting of at least three confusion lines along the tritan axis.

The electrooculogram was within the range of normal with an Arden ratio of 2.0 (SD 0.6). Amplitudes and latencies of the scotopic and photopic Ganzfeld ERG were normal in Parkinson patients.

Due to a reduced signal-to-noise ratio, the P-ERG could only be analyzed in four patients. In a healthy control group, the N35-P50 amplitude ratio of the $60^{\prime}$ and the $90^{\prime}$ check size was $1.06 \mu \mathrm{V}$ (SD 0.27). This compared well to Parkinson patients with a mean ratio of $1.03 \mu \mathrm{V}$ (SD 0.3).

P100 VEP latencies were normal (mean: 110 (SD $8.9 \mathrm{~ms}$ ). The N75-P100 VEP amplitude was slightly reduced by up to $3 \mu \mathrm{V}$ below the range of normal in three patients.

A multifocal ERG could be obtained in all eight patients. However, only four patients had an adequate signal-to-noise ratio permitting further analysis of the data, one of whom was later diagnosed with multi system atrophy (MSA). These patients had been treated with L-Dopa 250-500 mg/day which they had discontinued at least $12 \mathrm{~h}$ prior to examination. 


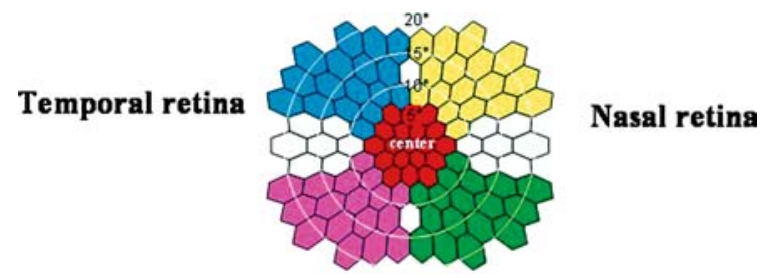

Figure 1. The areas of the response averages that were analyzed from the central $7.5 \mathrm{deg}$ and the four adjoining quadrants.

Figure 1 shows the areas of the response averages that were analyzed from the central $7.5 \mathrm{deg}$ and the four adjoining quadrants. These response averages were chosen, as a naso-temporal asymmetry of the mfOPs has previously been observed $[16$, 17]. Figure 2 shows the corresponding response averages. On average, the amplitude from the first negative trough (N1) to the first positive peak (P1) was slightly, but not significantly, lower in the central (Parkinson patients' mean: $32 \mathrm{nV} / \mathrm{deg}^{2}$, control mean: $46 \mathrm{nV} / \mathrm{deg}^{2}$ )) and nasal response averages of Parkinson patients. However, when the Parkinson patient's responses (3 bottom traces) and the patient with MSA (dashed line) were compared to four healthy volunteers (top 4 traces) no significant differences in waveform, amplitudes or latencies were observed. In particular, the oscillatory potentials at a latency of about 70-90 ms were present in all patients.

Figure 3 shows the same response averages shown in Figure 2, but with a different filter setting of $100-300 \mathrm{~Hz}$. The waveforms were filtered offline at $100-300 \mathrm{~Hz}$ in order to facilitate analysis of the oscillatory potentials. With this filter setting, OPs resulting from the m-sequence step stimulus can be visualized as well as those OPs resulting from the induced component, that is the stimulus in the sequence following the m-sequence step. Here, the most marked difference was observed in the nasal response average of the first OP. In the upper nasal field a mean amplitude of $1.3 \mathrm{nV} / \mathrm{deg}^{2}$ in patients compared to a mean amplitude of $1.93 \mathrm{nV} / \mathrm{deg}^{2}$ in the control group (P: 0.08). In the lower nasal field, the mean amplitude of $0.84 \mathrm{nV} / \mathrm{deg}^{2}$ in patients compared to a mean amplitude of $1.48 \mathrm{nV} / \mathrm{deg}^{2}$ in the control group (P: 0.08). Again there was no significant difference between the patients and healthy volunteers. OP-latencies also did not differ from normal. Enhancing the OPs by adding the OPs of the m-sequence step to those of the induced component failed to produce abnormal responses in the Parkinson patients.

Increasing the stimulus base interval to $106.6 \mathrm{~ms}$ also did not reveal retinal dysfunction in Parkinson patients other than a slight reduc-
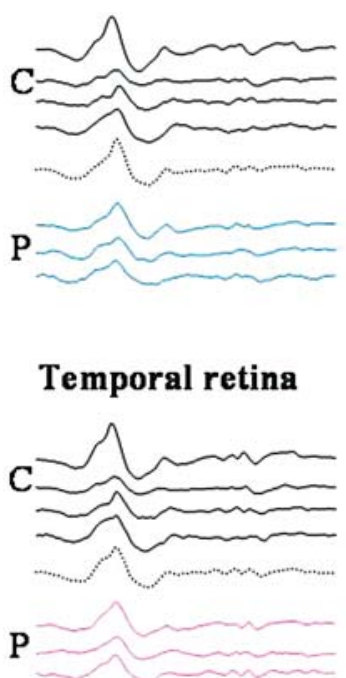

M3B Filter: 10-300 Hz

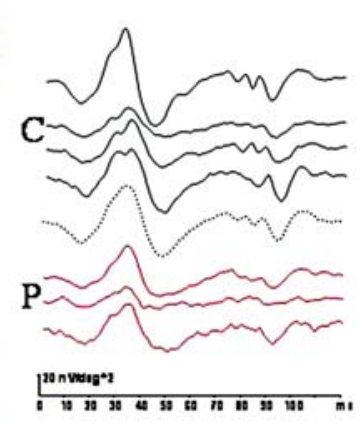

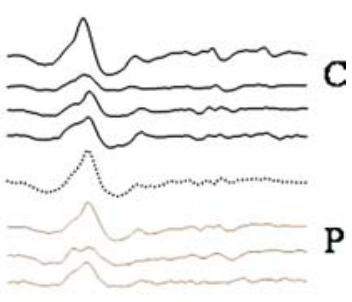

Nasal retina

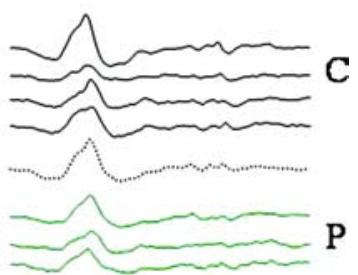

Figure 2. The responses resulting from the group averages shown in Figure 1 are depicted. For each response average, Parkinson patient's responses are shown in the three bottom traces, the patient with MSA is depicted by a dashed line, while for comparison, the waveforms of four healthy volunteers are also shown (top 4 traces). Note, that there are no significant differences in waveform. In particular, mf-oscillatory potentials are present in all patients. 


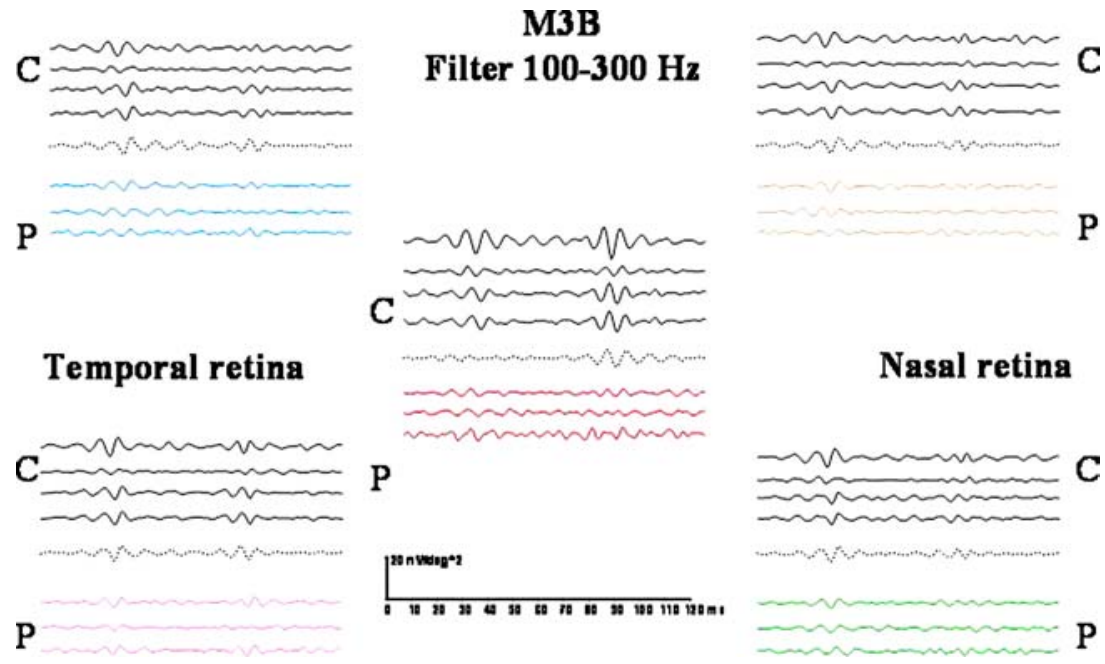

Figure 3. The same response averages shown in Figure 2, but with a different filter setting of $100-300 \mathrm{~Hz}$ to facilitate analysis of the oscillatory potentials. With this filter setting, OPs resulting from the M-sequence step stimulus are visualised between $20-50 \mathrm{~ms}$, as well as those OPs resulting from the induced component ( $70-90 \mathrm{~ms})$.

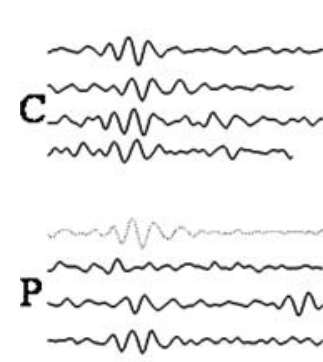

Temporal retina

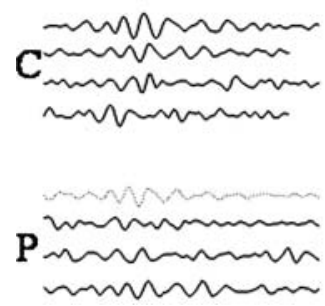

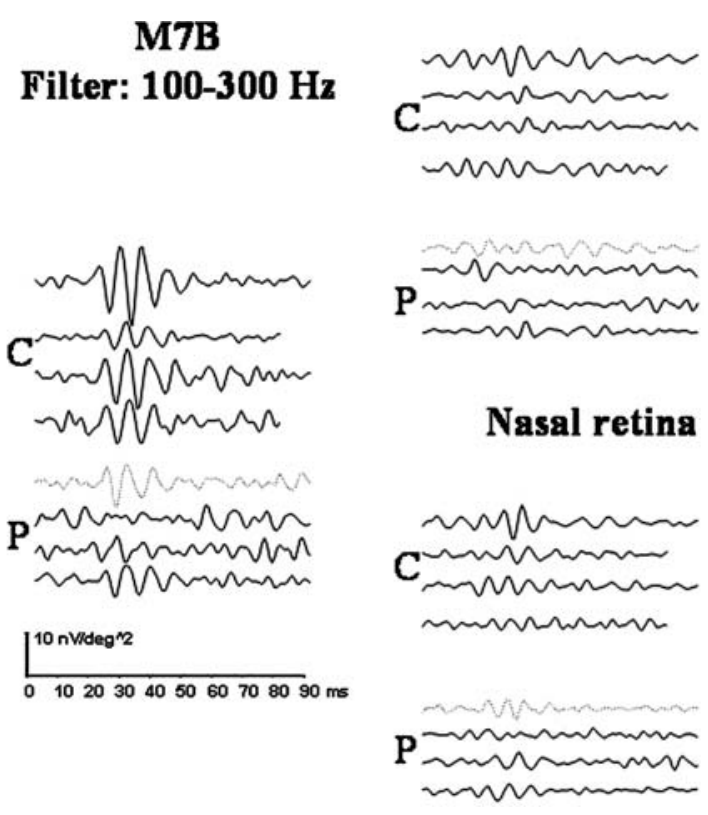

Figure 4. The same response averages for the M7B Stimulus with a filter setting of $100-300 \mathrm{~Hz}$ to facilitate analysis of the oscillatory potentials. With this stimulus sequence, an induced component is no longer apparent. For the same recording length, this stimulus sequence contains less signal than the M3B sequence. Central amplitudes of the OPs appear slightly reduced in Parkinson patients.

tion in the central amplitudes of the OPs in Parkinson patients. With this stimulus sequence, an induced component was no longer apparent (Figure 4). For the same recording length, this stimulus sequence contains less signal than the M3B sequence and therefore has a reduced signal-to-noise ratio.

\section{Discussion}

Our study shows that is possible to obtain mfERG recordings with a good signal-to-noise ratio in patients with moderate stages of Parkinson's disease. However, even in these early stages of disease, overlapping muscle artifacts resulted 
in a bad signal-to-noise ratio preventing further mfERG signal analysis in $50 \%$ of the patients.

While this pilot study included only a small number of patients, we nevertheless found a consistent presence of three mfOPs which is in contrast to our previous findings in NTG. Adjusting for the number of tests, electrophysiologic examinations showed no significant abnormalities in Parkinson patients. These negative results do not result from therapeutic dopamine substitution as L-dopa, which has a short half life of 2-3 h, had been discontinued at least $12 \mathrm{~h}$ prior to examination and these patients had been and were on no other medication.

Lack of dopamine in these patients with mild to moderate signs of Parkinson's disease seems to have little influence on the mfERG, and especially the mfOPs of the induced component under the stimulus conditions applied. Thus, dopaminergic amacrine cell dysfunction under these conditions may only have a minor influence on the mfERG recorded in moderate stages of Parkinson's disease. We cannot rule out, that differences might have become apparent had we been able to record more patients or more advanced stages of disease. In more advanced stages of Parkinson's disease an increased impairment due to motor symptoms such as tremor can be expected to render testing even more difficult.

Therefore, we feel that in order to further study the influence of dopaminergic amacrine cells on the mfERG, animal studies offer promising alternatives: systemic application of 1-methyl, 4-phenyl,1-2-3-6-tetrahydropyridine (MPTP) has been shown to decrease retinal dopamine content in primates [32]. Thus a possible animal model to examine the effect of dopamine depletion on the mfERG may be the application of MPDT. Indeed, in the primate model, MPDT has been shown to result in spatial frequency dependent changes in the PERG and PVEP [33]. Intravitreal application of this agent may allow for dose-effect analysis with the advantage of not inducing systemic motor symptoms such as tremor.

\section{References}

1. Palmowski AM, Sutter EE, Bearse MAJ, Fung W. Das Multifokale Elektroretinogram (MF-ERG) in der Diagnostik von Makula-Veränderungen am Beispiel der alters- abhängigen Makladegeneration (AMD). Ophthalmologe 1999; 96: 166-73.

2. Palmowski AM, Sutter EE, Bearse MAJ, Fung W. Das multifokale Elektroretinogram in der Diagnostik und Verlaufskontrolle lokalisierter Netzhauterkrankungen: Fallbericht eines Patienten mit Chorioretinopathia centralis serosa. Ophthalmologica 1999; 213: 327-35.

3. Bearse MA Jr, Suttter EE, Smith DN, Rose SJ. Early detection of macular dysfunction in the topography of the electroretinogram. In: America OSo, ed. Vision Science and its Applications 1. Santa Fe., Optical Society of America 1995: 318-21.

4. Kretschmann U, Ruther K, Usui T, Zrenner E. ERG campimetry using a multi-input stimulation technique for mapping of retinal function in the central visual field. Ophthalmic Res 1996; 28: 303-11.

5. Kretschmann U, Seeliger M, Ruether K, Usui T, Zrenner E. Spatial cone activity distribution in diseases of the posterior pole determined by multifocal electroretinography. Vis Res 1998; 38: 3817-28.

6. Palmowski AM, Sutter EE, Bearse MAJ, Fung W. Mapping of retinal function in diabetic retinopathy using the multifocal electroretinogram. Invest Ophthalmol Vis Sci 1997; 38: 2586-96.

7. Sutter EE, Bearse MAJ. The optic nerve head component of the human ERG. Vis Res 1999; 39: 419-36.

8. Sutter EE, Bearse MA, Jr. Extraction of a ganglion cell component from the corneal response. In: America OSo, ed. Vision Science and its Applications 1. Santa Fe, OSA 1995, 318-21.

9. Bearse M, Sutter EE, Smith DN, Stamper R. Ganglion cell components of the multi-focal ERG are abnormal in optic nerve atrophy and glaucoma. Invest Ophthalmol Vis Sci 1995; 36: S445.

10. Bearse MA, Sutter EE, Palmowski AM. New developments toward a clinical test of retinal ganglion cell function. In: America OSo, ed. Vision Science and its Applications 1. Washington DC, Optical Society of America, 1997, 280-3.

11. Bearse MAJ, Sutter EE, Palmowski AM. Luminancedependent enhancement of ganglion cell contributions to the human multifocal ERG. Invest Ophthalmol Vis Sci 1997; 38: S959.

12. Hood D, Frishman LS, Viswanathan S, Robson J, Ahmed J. Evidence for a ganglion cell contribution to the primate electroretinogram (ERG). Effects of TTX on the multifocal ERG in macaque. Vis Neurosci 1999; 96: 411-6.

13. Bearse MA Jr, Sim D, Sutter EE, Stamper R, Lieberman M. Application of the multi-focal ERG to glaucoma. Invest Ophthalmol Vis Sci 1996; 37: S511.

14. Bearse MA, Sutter EE, Shimada Y, Yong Y. Topographies of the optic nerve head component (ONHC) and oscillatory potentials (OPS) in the parafovea. Invest Opthaltmol Vis Sci 1999; 40: S17.

15. Palmowski-Wolfe AM, Allgayer, RJ, Vernaleken B, Schötzau A, Ruprecht KW. Slow-stimulation multifocal ERG in high and normal-tension glaucoma. Doc Ophthalmol 2006; In print.

16. Bearse MA, Shimada Y, Sutter EE. Distribution of oscillatory components in the central retina. Documenta Ophthalmologica 2000; 100: 188-205.

17. Rangaswamy NV, Hood DC, Frishman LJ. Regional variations in local contributions to the primate photopic flash ERG: revealed using the slow-sequence mfERG. Invest Ophthalmol Vis Sci 2003; 44: 3233-47. 
18. Wachtmeister L. Oscillatory potentials in the retina: what do they reveal. Prog-Retin-Eye-Res. 1998; 17(4): 485-521.

19. Arndt C, Derambure P, Defoort-Dhellemmes S, Hache J. Outer retinal dysfunction in patients treated with vigabatrin. Neurology 1999; 52(6): 1201-51999 Apr 12.

20. Horiguchi M, Suzuki S, Kondo M, Tanikawa A, Miyake Y. Effect of glutamate analogues and inhibitory neurotransmitters on the electroretinograms elicited by random sequence stimuli in rabbits. Invest Ophthalmol Vis Sci 1998; 39: 2171-6.

21. Bodis-Wollner I, Tzelepi A. The push-pull action of dopamine on spatial tuning of the monkey retina: the effects of dopaminergic deficiency and selective D1 and D2 receptor ligands on the pattern electroretinogram. Vis Res 1998; 38: 1479-87.

22. Djamgoz MB, Hankins MW, Hirano J, Archer SN. Neurobiology of retinal dopamine in relation to degenerative states of the tissue. Vision Res 1997; 37: 3509-29.

23. Langheinrich $\mathrm{T}$, Tebartz van Elst L, Lagreze WA, Bach M, Lucking $\mathrm{CH}$, Greenlee MW. Visual contrast response functions in Parkinson's disease: evidence from electroretinograms, visually evoked potentials and psychophysics. Clin Neurophysiol 2000; 111: 66-74.

24. Peppe A, Stanzione P, Pierantozzi M, Semprini R, Bassi A, Santilli AM, Formisano R, Piccolino M, Bernardi G. Does pattern electroretinogram spatial tuning alteration in Parkinson's disease depend on motor disturbances or retinal dopaminergic loss? Electroencephalogr Clin Neurophysiol 1998; 106: 374-82.

25. Nightingale S, Mitchell KW, Howe JW. Visual evoked cortical potentials and pattern electroretinograms in Parkinson's disease and control subjects. J Neurol Neurosurg Psychiatry 1986; 49: 1280-7.
26. Gottlob I, Weghaupt H, Vass C, Auff E. Effect of levodopa on the human pattern electroretinogram and pattern visual evoked potentials. Graefes Arch Clin Exp Ophthalmol 1989; 227: 421-7.

27. Skrandies W, Gottlob I. Alterations of visual contrast sensitivity in Parkinson's disease 1986; 5: 255-9.

28. Ikeda H, Head GM, Ellis CJ. Electrophysiological signs of retinal dopamine deficiency in recently diagnosed Parkinson's disease and a follow up study. Vision Res 1994; 34: 2629-38.

29. Muller T, Woitalla D, Peters S, Kohla K, Przuntek H. Progress of visual dysfunction in Parkinson's disease. Acta Neurol Scand 2002; 105: 256-60.

30. Palmowski AM, Berninger T, Allgayer R, HeinemannVernaleken B, Rudolph G. Effects of refractive blur on the multifocal electroretinogram. Doc Ophthalmol 1999; 99: 41-54.

31. Sutter EE, Tran D. The field topography of ERG components in man - I. The photopic luminance response. Vis Res 1992; 32: 433-46.

32. Ghilardi MF, Chung E, Bodis-Wollner I, Dvorzniak M, Glover A, Onofrj M. Systemic 1-methyl,4-phenyl,1-2-3-6tetrahydropyridine (MPTP) administration decreases retinal dopamine content in primates. Life Sci 1988; 43: 255-62.

33. Ghilardi MF, Bodis-Wollner I, Onofrj MC, Marx MS, Glover AA. Spatial frequency-dependent abnormalities of the pattern electroretinogram and visual evoked potentials in a parkinsonian monkey model. Brain 1988; 111: 131-49.

Address for correspondence: Anja M. Palmowski-Wolfe, University Eye Hospital, Mittlere Strasse 91, CH 4012, Basel Phone: +41-61-265-8722; Fax: + 1-61-265-8744

E-mail: PalmowskiA@uhbs.ch 